\title{
Automatic Unsupervised Landslide Detection Method Based on Single High-resolution Optical Image for Emergency Response
}

\author{
Xi Zhai, ${ }^{1}$ Wanzeng Liu, ${ }^{1 *}$ Chuan Yin, ${ }^{2}$ Yunlu Peng, ${ }^{1}$ Yong Zhao, ${ }^{\text {*** }}$ \\ Ying Yang, ${ }^{1}$ Xiuli Zhu, ${ }^{1}$ Ran $\mathrm{Li},{ }^{1}$ and Tingting Zhao ${ }^{1}$

\begin{abstract}
${ }^{1}$ National Geomatics Centre of China, 28 Lianhuachi West Road, Haidian District, Beijing 100830, China
\end{abstract} \\ ${ }^{2}$ School of Geomatics and Urban Spatial Informatics, Beijing University of Civil Engineering and Architecture, \\ 15 Yongyuan Road, Huangcun Town, Daxing District, Beijing 102616, China
}

(Received September 29, 2020; accepted November 17, 2020)

Keywords: urban landslide detection, high-resolution image, visual salience, reflective characteristics, morphological processing

Urban safety in mountainous areas is continuously seriously threatened by landslide disasters. Recently, many remote-sensing-based methods have been developed for landslide detection. However, many existing methods rely on multitemporal/multisource data, which require tedious data collection work and limit their practical capacity in real-time emergencies. Therefore, in this paper, we propose a novel unsupervised single-image-based landslide detection (USILD) method to automatically and quickly locate landslides and evaluate landslide risks, which can provide timely data for urban landslide responses. This method is designed to take full advantage of the visual salience and reflectance characteristics of landslides to produce a landslide risk map. Morphological processing is used to refine the final maps. The method is implemented and applied to the recent Ludian landslide event, in which hundreds of landslides occurred, on August 3, 2014. High-resolution satellite and aerial images obtained with sensor technology provided suitable experimental materials for this study. The experimental results show that our method can achieve higher accuracy and more automatic processing than other methods such as change detection using image differencing (CDD), change detection using ratio (CDR), $k$-means, and support vector machine (SVM). Moreover, the method requires no training samples and has a lower computational cost than supervised learning algorithms. Given the high detection accuracy and simple workflow, the proposed method is very promising for practical application in landslide emergency responses.

\section{Introduction}

Landslides, one of the most common natural hazards in mountainous and hilly areas, are triggered by extreme geological and climate events in mountainous areas, such as earthquakes, heavy rain, or volcanic eruptions, which can extensively damage the stability of the hard earth surface and cause many human casualties and property losses. ${ }^{(1)}$ Landslides seriously threaten

\footnotetext{
*Corresponding author: e-mail: lwz@ngcc.cn

** Corresponding author: e-mail: zhaoyong@ngcc.cn

https://doi.org/10.18494/SAM.2020.3131
} 
the safety of mountainous urban areas, with thousands of casualties directly resulting from landslides from 2004 to 2010 . $^{(2)}$ To protect lives and property, it is critical to effectively monitor landslides. Quickly locating landslides and identifying their intensity and spatial distribution can be extremely useful for making instant decisions and coordinating rescue efforts in emergency responses. Many methods have been proposed and remote sensing is one of the major supporting techniques. With its wide coverage, short revisiting cycle, and easy data access, remote sensing has attracted a lot of attention in recent years and has become one of the major means of landslide mapping and management. ${ }^{(3)}$

Owing to the progress of earth observation sensor technology, satellite images can be taken from satellite sensors, such as QuickBird and Landsat, and aerial images can be taken from airborne sensors, such as BlueSky, Sanborn, IKONOS, and SPOT5 sensors. Through the fusion of several commercial sensor images, the spatial resolution can reach about $0.5-1 \mathrm{~m}$. These high-resolution images provided suitable experimental materials for this study. Satellite remote sensing, including passive optical remote sensing and active microwave remote sensing, provides ideal data resources for studying landslides. ${ }^{(4)}$ Time series of satellite imagery record a massive amount of information that could be valuable for landslide analysis. Since the 1970s, when Landsat was launched into space, satellite images have been studied for landslide mapping. ${ }^{(5)}$ In recent years, many advanced remote sensing techniques, such as high-resolution satellites, lidar, and unmanned aerial vehicles (UAVs), have been applied to landslide studies, greatly enhancing the hazard monitoring and emergency abilities of the relevant authorities.

Many landslide detection methods have been proposed and assisted the conduction of operational landslide investigations. However, most of the methods require multitemporal/multisource images and take considerable time for data preprocessing, e.g., image retrieval, image registration, and image rectification, which largely limits their practical application in real-time emergencies. Also, frequent cloud cover significantly increases the challenges of collecting usable data at regular time intervals. Advanced intelligent analysis of a single image could help avoid these issues and has great potential to improve the timeliness and effectiveness of responding to landslide emergencies.

Considering the problems caused by the lack of various materials in emergencies, i.e., the difficulty of accessing multitemporal/multisource images, we propose an efficient unsupervised single-image-based landslide detection (USILD) method to rapidly locate landslide sites and calculate hazard areas to provide timely data for assisting hazard response decision making. This method is specifically designed for emergencies and only needs a single image to automatically locate landslides. The USILD method is the first to introduce visual salience in the computer vision field to landslide detection and takes full advantage of the salience index from a frequency-tuned (FT) model and a landslide index (LI) obtained from the reflected characteristics to produce a preliminary landslide risk map. Then, morphological processing is applied to obtain a final refined landslide map, which shows the disaster area. The proposed method is used to investigate the Ludian landslide event, which involved hundreds of landslides caused by a large earthquake that occurred in Ludian County, Yunan Province, China, on August 3, 2014, some of which occurred near urban suburbs. The experimental results show that the proposed method can achieve higher accuracy and more automatic processing than other 
methods such as change detection using image differencing (CDD), change detection using ratio (CDR), $k$-means, and support vector machine (SVM). Moreover, the method requires no training samples and has a lower computational cost than supervised learning algorithms. In a disaster emergency, owing to the difficulty of data access and the complexity of data processing, it is very challenging to obtain first-hand information in a short time to support a quick response. Owing to its low data requirement and high efficiency, the proposed method can play an important role in landslide disaster emergencies by quickly and automatically locating the landslide sites. Given the high detection accuracy and simple workflow, the proposed method is very promising for practical applications in landslide emergency responses.

The rest of this paper is organized as follows. Section 2 introduces related work. Section 3 introduces the research area and materials. Section 4 introduces the USILD method in detail. In Sect. 5, the experimental results are provided and a detailed discussion is given. Section 6 concludes this work and gives our future work.

\section{Related Work}

In the past decade, many remote-sensing-based landslide detection algorithms have been proposed. ${ }^{(6)}$ These methods can be roughly divided into three main categories: (1) Change detection methods. Changes in land cover or reflective/radiometric properties are good indicators of landslides and can be automatically calculated by comparing image time series. Therefore, many landslide studies based on different change detection methods have been carried out, including post-classification comparison methods, temporal image differencing, and Bayesian probabilistic methods. For example, Lu et al. presented a semiautomatic approach using object-oriented change detection for landslide mapping with high-resolution optical images. ${ }^{(7)} \mathrm{Li}$ et al. proposed a change detection approach using a Markov random field method and aerial orthophotos to map landslides. ${ }^{(8)}$ Recently, Uemoto et al. developed a new landslide detection method using pre- and post-event airborne X-band synthetic aperture radar (SAR) to calculate landscape height and amplitude differences. ${ }^{(9)}$ Handwerger et al. used the Google Earth Engine platform and SAR amplitude change detection to locate landslides. ${ }^{(10)}$ (2) Digital elevation model (DEM)-based methods. A DEM describes the topography information of the study area; through analysis of the deformation and displacement of the surface topography, landslide sites can be effectively located. These methods include interferometric SAR (InSAR)-based techniques, lidar-based techniques, and UAV-based techniques. For example, Shi et al. retrieved three-dimensional landslide surface displacements based on the analysis of time series InSAR data. ${ }^{(1)}$ Haneberg et al. conducted landslide mapping and modeling using high-resolution lidar data. ${ }^{(12)}$ Ohki et al. detected landslides in mountainous regions based on polarimetry and interferometric coherence. ${ }^{(13)}$ (3) Multisource/multitechnique-based methods. These methods take advantage of different remote sensing data or techniques to better map landslides. For example, Barlow et al. detected landslides using Landsat enhanced thematic mapper plus (ETM+) images and DEM data. ${ }^{(14)}$ Plank et al. mapped landslides based on change detection with optical images and polarimetric SAR data in vegetated areas. ${ }^{(15)}$ Pradhan et al. proposed an automatic landslide detection method by integrating object-based analysis and 
random forest classification with lidar and QuickBird images. ${ }^{(16)} \mathrm{Xu}$ et al. monitored the Gold Basin landslide by multisource remote sensing and numerical models. ${ }^{(17)}$

Machine learning and AI techniques are also adopted to pursue advanced intelligent landslide detection and further improve the detection accuracy. ${ }^{(18)}$ For example, Cheng et al. proposed an automatic landslide detection method through scene classification based on a bag-of-visual-words (BoVW) model and a probabilistic latent semantic analysis (PLSA) model. $^{(19)} \mathrm{Wu}$ and Chang used back-propagation neural networks based on a lidar DEM to classify landslides. ${ }^{(20)}$ Ding et al. proposed an automatic landslide recognition method based on a convolutional neural network $(\mathrm{CNN})$ and texture change detection. ${ }^{(21)}$ Ghorbanzadeh et al. compared the performances of different machine-learning-based methods including deep CNNs. ${ }^{(22)}$ Yu et al. proposed a contour-based deep learning framework for landslide detection in Nepal. ${ }^{(23)}$ However, this framework is still under development and much more work is needed to train the AI models to learn the knowledge about landslides. Currently, the application of AI in practical landslide warning, monitoring, and responding is still in the exploration stage.

\section{Study Area and Materials}

\subsection{Study area}

In this study, we focus on a typical landslide: the Ludian landside event, which occurred in Ludian County, located in the northeast of Yunnan province, south of Zhaotong City, and on the north bank of the Niulan River (Fig. 1). The county area is $1519 \mathrm{~km}^{2}$, of which $87.9 \%$ is mountainous and $12.1 \%$ is plain.

At $16: 30$ on August 3, 2014, a 6.5-magnitude earthquake hit Ludian County $\left(27.1^{\circ} \mathrm{N}, 103.3^{\circ} \mathrm{E}\right)$. A high density of faults has developed in the earthquake area, and most of the faults are active. The regional ground stress is high and abnormal, seismic activity is frequent, rocks are broken, the geology is complex, and many landslides are induced by earthquakes.

\subsection{Data acquisition and collection}

It is difficult to obtain cloud-free satellite images over mountainous areas owing to high moisture, particularly during heavy rains and earthquakes. ${ }^{(17)}$ For the Ludian landslide, a high-resolution Google image from August 20, 2014 was used for post-event landslide investigation with a pre-event image from December 6, 2011 for comparative experiments. These images are from Google Earth and are not from a single data source, but are the integration of satellite images and aerial images. Part of the satellite images were taken from the QuickBird and Landsat sensors, while aerial images were taken from the BlueSky, Sanborn, IKONOS, and SPOT5 sensors. Through the fusion of several commercial satellite images, the spatial resolution can reach about 0.5-1 m. For the Ludian landslide event, level 19 (L19) images at a spatial resolution of $0.6 \mathrm{~m}$ were utilized to locate multiple landslides. 


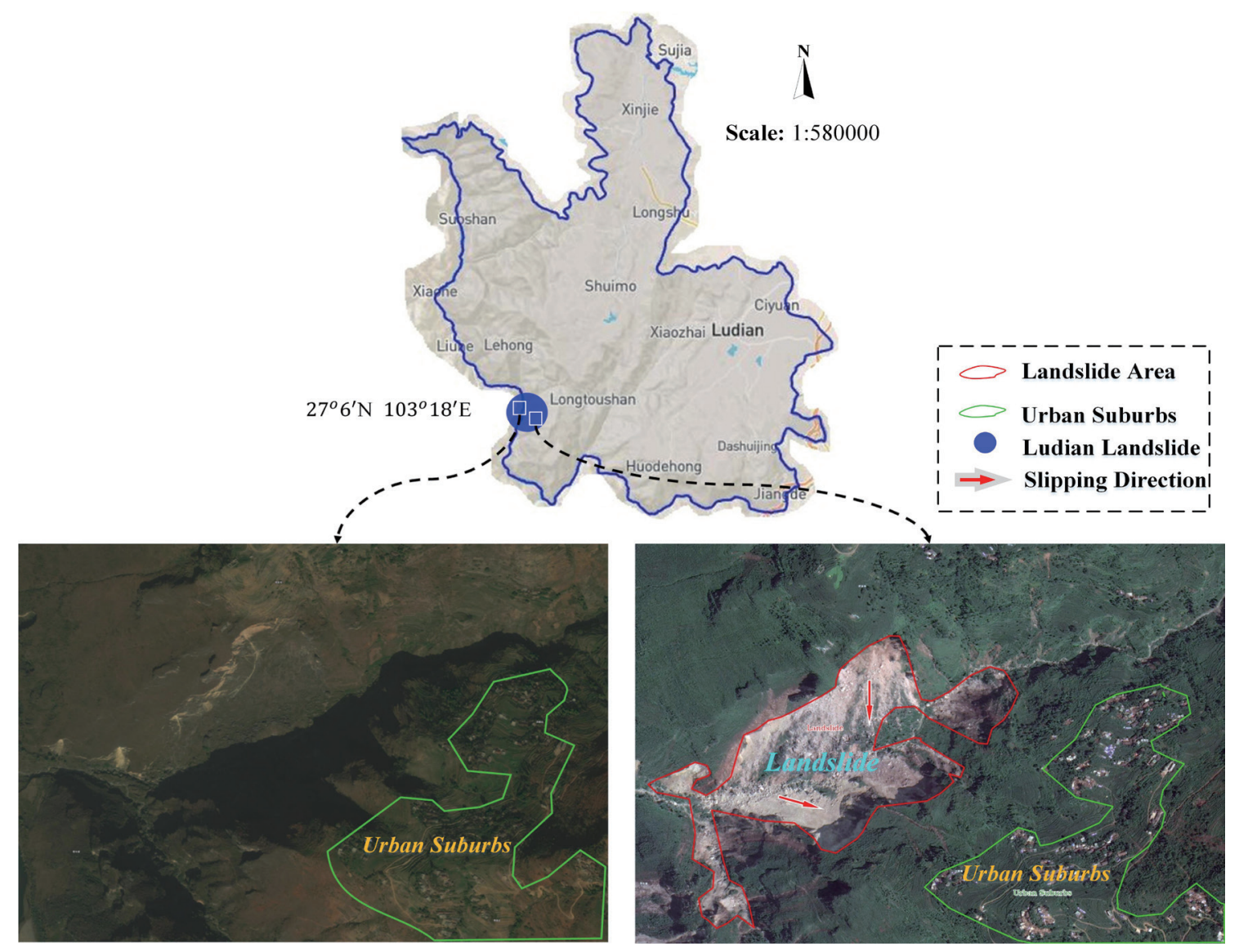

Fig. 1. (Color online) (Upper) Geographical location of the study area. (Lower left) Pre-event Google image showing part of the area of the Ludian landslide event acquired on December 6, 2011. (Lower right) Post-event Google image of the same area acquired on August 20, 2014.

\section{Methods}

The overall workflow of the proposed USILD method consists of three major steps, as shown in Fig. 2. First, preliminary preprocessing is performed on the high-resolution image acquired, including panchromatic sharpening, region of interest (ROI) tailoring, thin cloud removal, and background suppression. Second, a landslide risk map is automatically produced in an unsupervised manner from the single image by evaluating the visual salience and LI automatically via an estimation model. Third, morphological processing is conducted to obtain the final refined landslide detection map.

\subsection{Data preprocessing}

Data preprocessing is the preliminary task for landslide investigation. In this study, we adopted a general data preprocessing framework. For the Ludian landslide, as only an RGB Google image was available, this panchromatic fusion step was ignored. Then, considering 


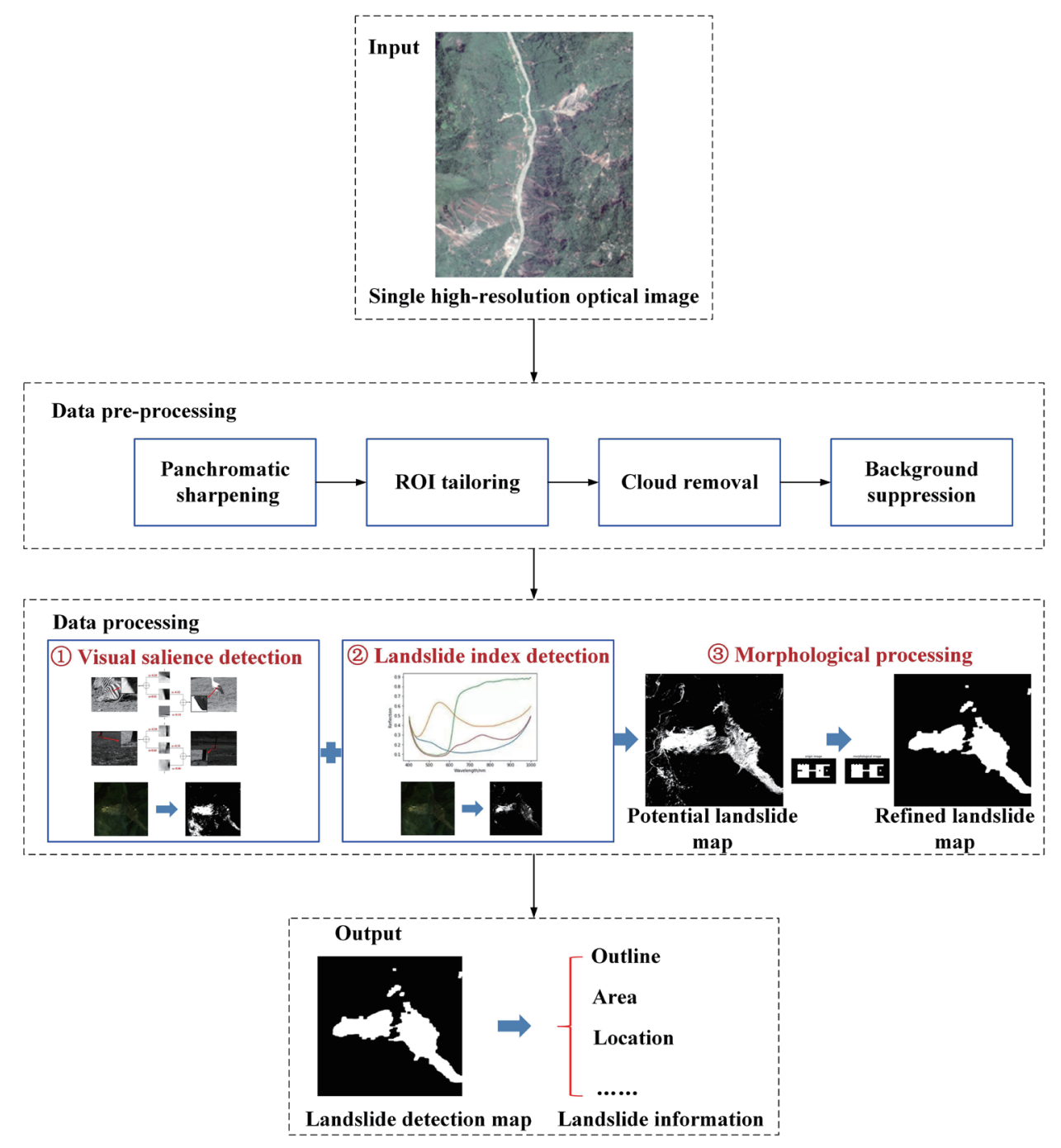

Fig. 2. (Color online) Overall technical route of this study.

that the landslide sites only take up a small portion of the whole area and are easily obscured by background noise, the original images were generally tailored to produce ROI image batches to reduce the computational complexity according to the post-event reported geographic locations of the landslides.

For the Ludian landslide, part of the post-event Google image was contaminated by thin clouds that block part of the area and prevent our analysis of the blocked or shadowed areas. Therefore, it was very desirable to remove the thin clouds. In this study, the well-known dark channel prior (DCP) method was used to remove the thin clouds in the image.

The DCP method is an effective haze removal and depth map estimation method for outdoor RGB images and is widely used for thin cloud removal from remote sensing images. ${ }^{(24)}$ This method is based on the atmospheric scattering model and is formulated as

$$
I(x)=J(x) t(x)+A(1-t(x)),
$$


where $I(x)$ denotes the acquired remote sensing image with thin clouds, $J(x)$ denotes the cloudfree image to be recovered, $t(x)$ denotes the transmission map, $x$ is the pixel location, and $A$ is the global atmospheric light, which is generally set as a constant in practice. ${ }^{(24)}$

This method assumes that the dark channel values of the hazy image are mainly contributed to by path radiance/air light; therefore, the dark pixels can be used for haze transmission estimation. ${ }^{(24)}$ The DCP can be obtained by searching for the minimum digital number (DN) values in the red, green, and blue channels within a local patch with a size of $5 \times 5$ for each pixel. In this research, the search for local patches is simplified by using a pixel search to improve efficiency. Then, the transmission map $t(x)$ is calculated with the dark channel obtained. Finally, the cloud-free image is recovered through the atmospheric scattering model in Eq. (1), as shown in Fig. 3(c).

In general, landslide areas are brighter than non-landslide areas, which is commonly due to changes in the land surface from vegetation or other types of natural cover to bare rocks or bare soil in landslide areas. To highlight this characteristic to improve the visual salience detection, we further suppressed the background by subtracting certain values from the DN value of each pixel according to the statistical characteristics of the $\mathrm{DN}$ values in each channel. For the Ludian landslide study, the background suppression was conducted by subtracting 65, 65, and 55 from the DN values of each pixel for the red, green, and blue channels, respectively.

\subsection{USILD method}

Considering that only a single temporal RGB image is available for landslide detection, timeseries information is unavailable. On the other hand, considering that it is time-consuming to label samples for landslide learning, in this study, an USILD method is presented to rapidly locate landslide sites. This method fully takes advantage of the visual significance and reflective properties of landslides to produce a coarse preliminary map, and with morphological processing, a final refined detection map is obtained.

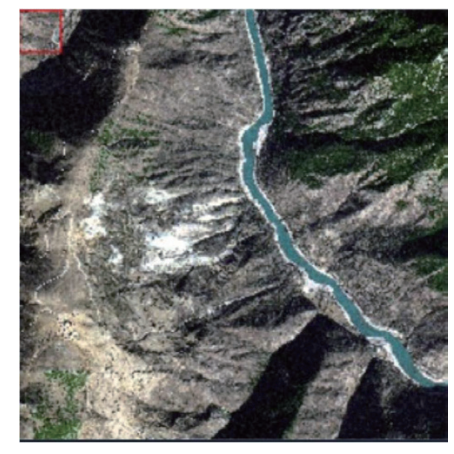

(a)

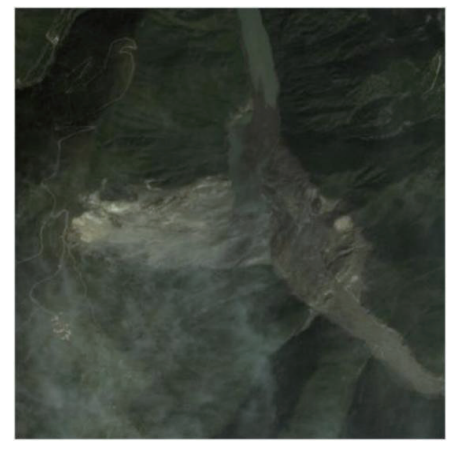

(b)

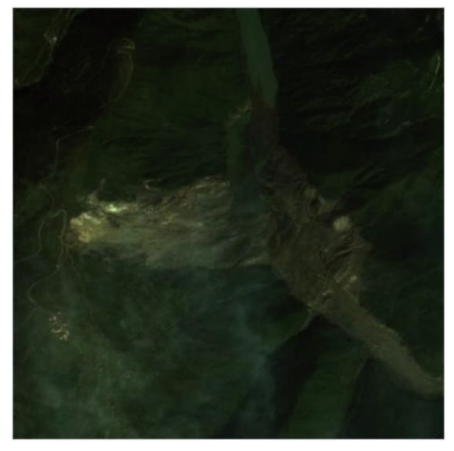

(c)

Fig. 3. (Color online) Thin cloud removal based on the DCP method: (a) pre-event image, (b) post-event image, and (c) cloud-free post-event image. 


\subsubsection{FT visual salience detection}

Based on the fact that landslides generally lead to significant land-cover changes, i.e., from vegetation or other natural surfaces to bare rocks or bare soil, landslide areas differ from the surroundings. Therefore, it is reasonable to introduce a visual significance scheme to extract potential landslide areas. In this research, an efficient FT salient region detection method (FT method) is implemented. ${ }^{(25)}$

The FT method is a well-known visual salience detection method in the computer vision field. It is based on the assumption that an image can be divided into a low-frequency component and a high-frequency component in the frequency domain, with the low-frequency component reflecting the overall information of the image, such as the outline of an object and the basic composition areas, and the high-frequency component reflecting the detailed information of the image, such as the texture of an object. ${ }^{(25)}$ The FT method focuses more on the low-frequency information to detect the salient areas. In practice, this method uses a Gaussian smoothing window sized to cancel the highest frequency. Then, fully exploiting the advantages of the CIELAB color space, the FT method detects the salient regions through color space conversion from RGB space to CIELAB color space and calculates the salience of each pixel by the following formulation:

$$
S(x)=\left\|I_{\mu}-I_{W h c}(x)\right\|_{2}^{2},
$$

where $I_{\mu}$ denotes the mean image feature of a pixel and $I_{W h c}(x)$ represents the corresponding CIELAB color space feature of the pixel after Gaussian blurring. The CIELAB color space contains three main components, $L, a$, and $b$, with $L$ denoting the luminance component and $a$ and $b$ denoting the color components, and reflects the properties of land objects from different perspectives. This color space is reasonably in line with human visual mechanisms.

In general, the FT method has the following five characteristics:

1. Emphasizes the largest salient objects.

2. Uniformly highlights whole salient regions.

3. Establishes well-defined boundaries of salient objects.

4. Disregards high frequencies arising from texture, noise, and blocking artifacts.

5. Efficiently outputs full-resolution saliency maps.

Therefore, in this research, we implement the FT method to extract pixels from areas with the high potential of a landslide.

\subsubsection{LI construction}

To better indicate the areas at risk of a landslide, we construct a simple LI based on the reflective characteristics to assist in visual salience detection and produce a more accurate risk map. Since landslides generally markedly change the natural surface, i.e., vegetation is converted into bare rocks or bare soil, landslide areas generally consist of bare rocks and 
bare soil, which have different reflective characteristics from the surrounding background. Therefore, it is natural to construct spectral indices to assist in landslide analysis.

As only RGB bands are available, the spectral information is very limited. Considering the reflective differences between vegetation and bare rock/bare soil, the LI is constructed as follows:

$$
L I(x)=\|b(x)-g(x)\|_{2}^{2}+\| b(x)-\left.r(x)\right|_{2} ^{2},
$$

where $b(x), g(x)$, and $r(x)$ denote the DN values of the blue, green, and red channels, respectively. The LI comprehensively considers the reflective characteristics of different land objects in each channel and has some ability to indicate potential landslide areas.

As the visual salience detection and LI detection are based on different mechanisms, the landslide risk maps produced can provide complementary information that improves the detection accuracy. Therefore, in this research, these two risk maps were synthesized to comprehensively use the complementary information to obtain a better indicator map, as in the following formulation:

$$
P(x)=S(x) \otimes L I(x),
$$

where $\otimes$ denotes the point multiplying operation with the corresponding elements multiplied. Through multiplication, the potential landslide pixels with high confidence can be further enhanced, with the potential landslide pixels with low confidence effectively weakened. In this way, a more accurate risk map can be obtained. In general, by taking advantage of the visual salience and LI, the potential landslide areas in the image can be clearly indicated.

\subsubsection{Morphological processing}

The detection map produced above is relatively coarse, and a certain amount of interference from similar land objects is inevitably included, such as buildings, roads, and bare soil. Therefore, morphological processing is implemented to postprocess the potential map and refine the detection result.

Morphological processing is a powerful tool for feature extraction or image postprocessing and is widely used in the remote sensing field. ${ }^{(26)}$ This type of processing consists of four main operations based on specific structural basic units, opening, closing, eroding, and dilating, with each operation having a different purpose. ${ }^{(26)}$

Specifically, for the landslide study, the eroding operation is first implemented to exclude the noise and outliers in the risk map. Line erosion with specific angles is used for specific regions of the risk map to remove the interference from linear objects, such as roads. A water index is constructed to remove the interference from the river by subtracting the red band of the Google image from the green band, where the pixels with a value greater than 0 are water pixels. Then, the closing operation with a specific structural basic unit, i.e., $(35,35)$, is used to fill the holes 
within the landslide areas. Finally, a median filter with the basic unit of $(25,25)$ is used to further remove the noise and outliers.

After a series of morphological processes, a refined detection map with less noise and fewer outliers is obtained. Then, through binarizing with an empirical threshold, i.e., 150, the final landslide detection map is obtained.

\section{Results and Discussion}

\subsection{Results}

\subsubsection{Experimental settings}

The proposed USILD method was implemented for the Ludian landslide in 2014 using the high-resolution Google image from August 20, 2014. The Ludian landslide had hundreds of inconspicuous landslide regions, presenting a very challenging detection task. Reference maps of the Ludian landslide produced by three experienced experts through visual interpretation were collected as the ground truth, as shown in Fig. 4(a). To verify the effectiveness of the

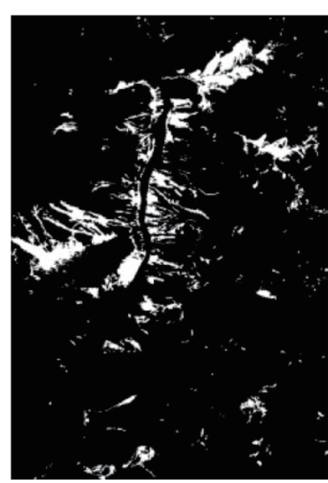

(a)

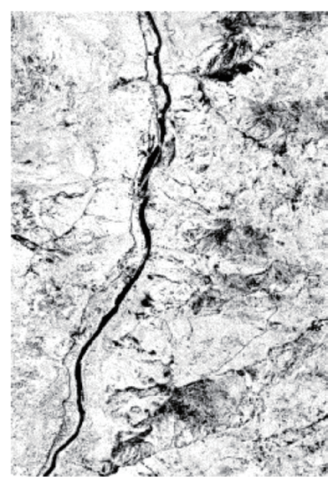

(d)

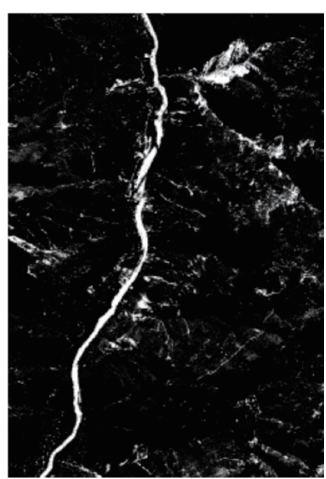

(b)

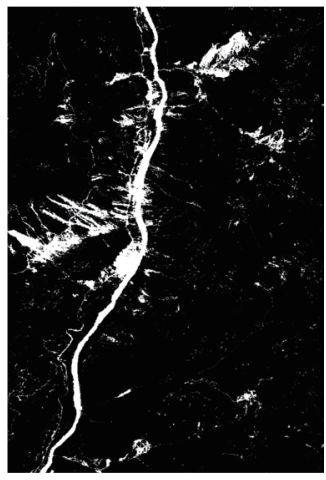

(e)

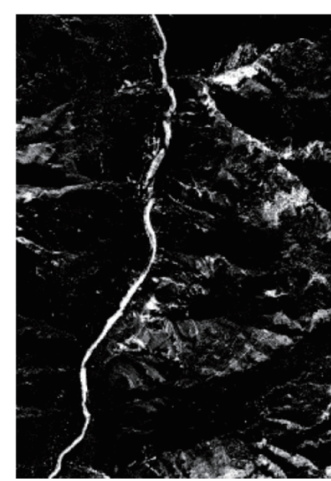

(c)

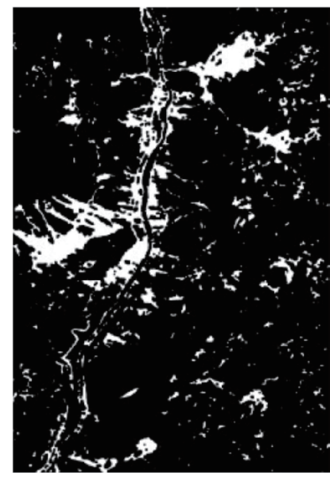

(f)

Fig. 4. Landslide identification results of different methods for the Ludian landslide: (a) ground truth, (b) CDD method, (c) CDR method, (d) PCA $k$-means method, (e) SVM method, and (f) proposed USILD method. 
proposed method, four representative landslide detection methods were selected as comparative methods, including single-image SVM classification, ${ }^{(27)} \mathrm{CDD},{ }^{(28)} \mathrm{CDR},{ }^{(29)}$ and change detection based on PCA $k$-means. ${ }^{(30)}$ The first method is a supervised method, and the other methods are unsupervised methods that use the DN values of the image as the input. In addition, as the last three comparative methods are based on multiple images, an accurate geometric correction based on a polynomial model with 30 ground control points was applied to images before landslide detection, with root mean square errors (RMSEs) of less than 0.5 pixels for the preevent and post-event images.

In the experiments, all of the methods were implemented in MATLAB code in a Windows 10 environment with a 64 Intel(R) Xeon(R) Silver $4110 \mathrm{CPU}(2.10 \mathrm{GHz})$ and $16 \mathrm{~GB}$ of memory without any parallelization technology. The results reported are averages of five experiments. To comprehensively compare the performances of different methods, both qualitative and quantitative evaluations are given. The common quantitative metrics of overall accuracy (OA), Kappa, producer accuracy (PA), and user accuracy (UA) were used in this study, and the time cost of each method is provided.

\subsubsection{Experimental results and analysis}

For the Ludian landslide, the landslide identification maps of different methods are shown in Fig. 4, with the corresponding quantitative evaluations given in Table 1. The figure and the table show that the proposed USILD method displays optimal or suboptimal landslide detection performance with very low time costs for this landslide scene, which suggests its effectiveness for landslide emergencies.

Specifically, owing to serious disturbances from similar land materials and large changes in land cover, the three change-detection-based methods performed badly for both landslide scenes and failed to effectively distinguish the landslide areas from the background, with a large number of omissions and false detections in the detection results. As a result, the detection precision of these three methods was low. The PCA $k$-means method had the worst performance: nearly the whole scene except the river was mistakenly identified as landslide areas. Consequently, PA of only $62.12 \%$ and UA of only $5.94 \%$ were obtained. Compared with the change-detection-based methods, the single-image SVM classification method performed much better, obtaining detection results with a much higher OA of $90.06 \%$. With the help of

Table 1

Quantitative evaluations of identification results of different methods for the Ludian landslide.

\begin{tabular}{ccccccc}
\hline Method & Class & CDD & CDR & PCA $k$-means & SVM & USILD \\
\hline \multirow{2}{*}{ PA (\%) } & Landslide & 32.54 & 24.96 & 62.12 & $\mathbf{8 3 . 1 1}$ & $\underline{79.15}$ \\
& Non-landslide & 95.21 & 92.33 & 17.89 & $\underline{90.64}$ & $\mathbf{9 4 . 9 8}$ \\
\hline \multirow{2}{*}{ UA (\%) } & Landslide & 36.18 & 21.37 & 5.94 & $\underline{42.57}$ & $\mathbf{5 6 . 8 4}$ \\
& Non-landslide & 94.42 & 93.65 & 84.98 & $\mathbf{9 8 . 4 7}$ & $\underline{98.20}$ \\
\hline \multicolumn{2}{c}{ OA (\%) } & 90.38 & 87.14 & 21.30 & $\underline{90.06}$ & $\mathbf{9 3 . 7 6}$ \\
Kappa & 0.0198 & 0.1606 & -0.0375 & $\underline{0.5134}$ & $\mathbf{0 . 6 2 8 3}$ \\
Time (s) & 6.3 & 6.3 & $2.3 \times 10^{3}$ & 301 & 40.2 \\
\hline
\end{tabular}


supervised information and labeled samples, this method could identify the landslide areas well with few omissions. This method maintained the details and outlines of the landslide areas well. However, many roads and buildings were misclassified as landslide areas owing to the high similarity between these features, which led to a higher false detection rate. Consequently, the UA of this method was only $42.57 \%$.

Compared with the above methods, the proposed USILD method yielded good performance for the Ludian landslide locations. The areas identified were visually consistent with the original image and ground truth, with major parts of the landslides effectively located and noise and outliers removed. This method obtained the highest accuracy with OA of $93.76 \%$ and Kappa of 0.6283 , suggesting its effectiveness. Despite the very challenging task of identifying hundreds of small landslide regions, most of the landslide areas were accurately identified.

Figure 5 presents a more intuitive comparison of the performances of different methods, from which similar conclusions can be drawn. The three change-detection-based methods had the worst performance with low detection accuracies. The single-image SVM-classification-based method detected the landslides well due to the supervised information. However, it is time-consuming to acquire training samples, making it unsuitable for emergencies. The proposed USILD method showed a performance comparable to that of the SVM method, but in an unsupervised manner, which further illustrates the effectiveness and superiority of this method. In emergency applications, the proposed unsupervised USILD method is expected to perform slightly better than the supervised SVM method when the training samples are very limited or there are no training samples, which further shows its effectiveness.

From the perspective of time cost, the proposed USILD method can quickly locate the landslide areas. From Table 1, although the SVM method could locate the landslides with the help of training samples, the time cost was very high. Also, collecting high-quality training samples consumes large amounts of labor and time, which limits the capability of this method

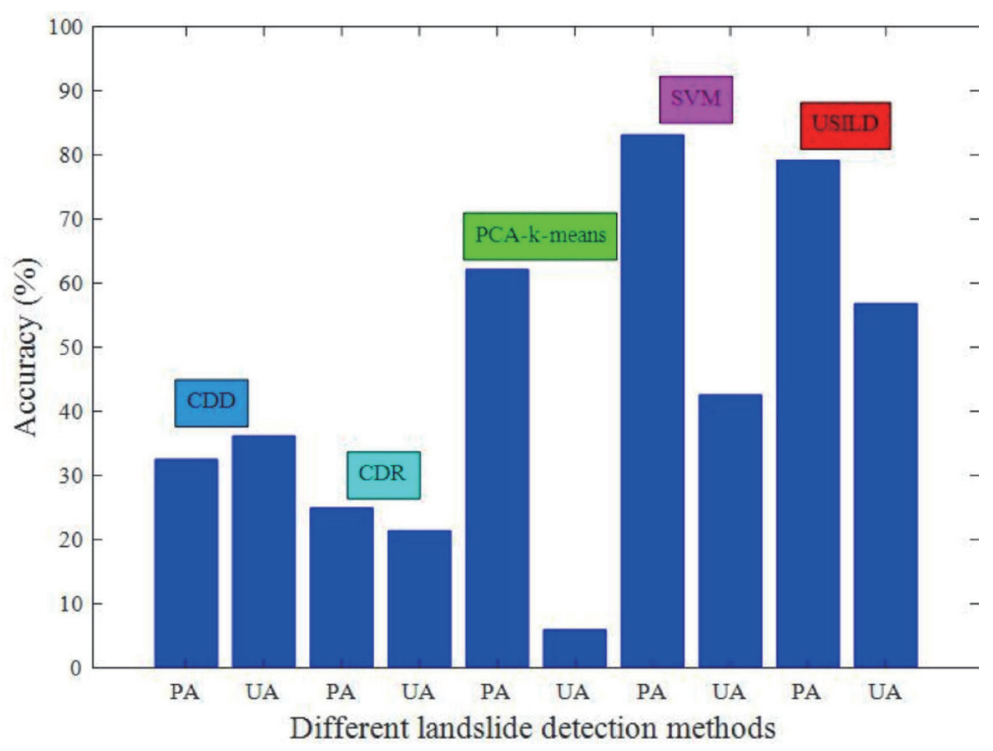

Fig. 5. (Color online) Comparisons of different methods for identifying Ludian landslide. 
for emergencies to a large degree. The proposed method can obtain accuracy comparable to that of the SVM method in much less time, which further suggests its effectiveness and capability for landslide emergencies.

\subsubsection{Landslide area estimation}

The landslide area is an important indicator for estimating the magnitude of landslides and hazard assessments and is widely used in landslide emergencies. ${ }^{(31)}$ In this study, we estimated the Ludian landslide area according to the detection result. In practice, the area can be regarded as a result derived from the detection map and can be estimated using the following formula:

$$
\text { Landslide area }=\# \text { landslide pixels } \times 2^{2} \times 10^{-6} \text {, }
$$

where \# denotes the number of landslide pixels, 2 corresponds to the spatial resolution of the image, and the unit of the landslide area is $\mathrm{km}^{2}$.

According to the landslide detection map, a risk landslide area of approximately $23.56 \mathrm{~km}^{2}$ occurred during the Ludian landslide event. This figure is close to those in official reports, which means that the proposed USILD method can provide technical support for hazard assessment.

\subsection{Discussion}

A landslide emergency requires the rapid location of landslide areas and the accurate assessment of the hazard level. The experimental results show that the three change-detectionbased methods had worse performance than our proposed method for landslide identification, with poor maps and low precision for landslide scenes, and the landslide area derived from the detection map was not reliable. As a result, these methods will not be effective for landslide responses. Compared with these three methods, the SVM method had much better performance. Owing to the supervised information and labeled samples, the SVM method accurately located the landslide areas. However, this method is based on labeled samples, and the labeling process generally takes a long time, which limits the applicability of this method in emergencies to a large degree.

In general, the proposed USILD method can locate landslide areas well at a low time cost. This method obtained an OA of $93.72 \%$ for the Ludian landslide, which means that over $93 \%$ of the pixels were correctly identified. Both the omission rate and the false detection rate were acceptable. A landslide risk area was evaluated from the detection map for the Ludian landslide, which is very important for contingency plans. The total time required for the proposed USILD method was approximately $40 \mathrm{~s}$ for the Ludian landslide. These results mean that the USILD method can efficiently locate landslide areas, which can provide the initial data for emergencies to support decisions.

To evaluate the effectiveness of the landslide risk estimation by the proposed USILD method, the risk maps produced by visual salience detection and land index detection for the Ludian 
landslide are respectively shown in Figs. 6(b) and 6(c). The synthesized risk map based on the above two maps is shown in Fig. 6(d). The figures show that both the visual salience detection and land index detection have certain capabilities for indicating landslides. Most of the landslide areas were detected well in the risk maps, although a certain amount of interference from objects with similar background objects was included.

This result suggests that visual salience can well capture the salient areas with obvious hue differences in the surroundings caused by landslides, and the land index can well indicate landslides based on the reflective differences caused by land cover changes. The synthesized landslide risk map better indicates landslides by taking advantage of the complementary information from these two detection methods. Through morphological processing of the risk map, a refined landslide detection map can be obtained, as shown in Fig. 6(e). In the noise removal process, certain landslide areas are removed and the edges of landslide areas are smoothed. Therefore, the noise and detection rate need to be balanced in the practical use of this technique.

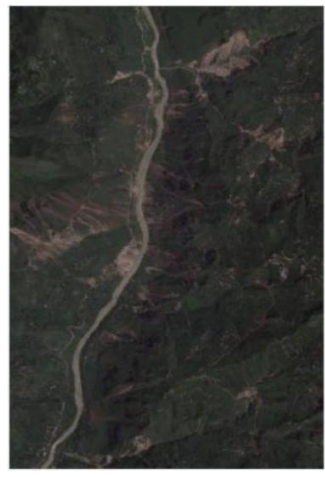

(a)

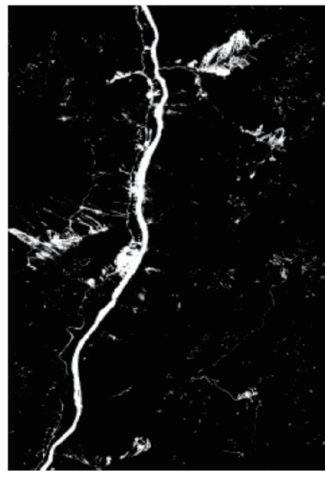

(b)

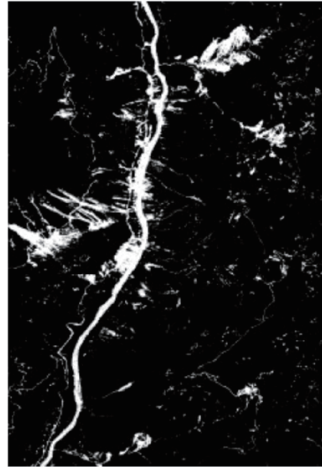

(c)

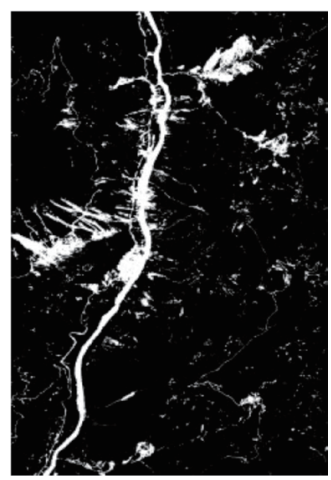

(d)

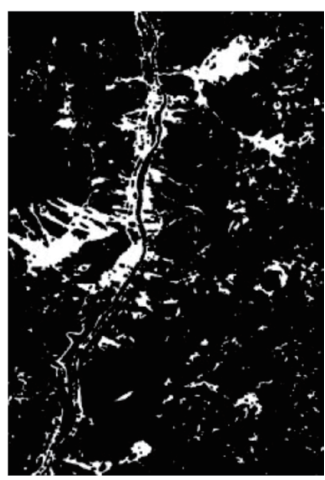

(e)

Fig. 6. (Color online) Risk maps produced by the proposed USILD method for the Ludian landslide: (a) postevent Google image scene, (b) risk map produced by visual salience detection, (c) risk map produced by land index detection, (d) synthesized landslide risk map, and (e) landslide detection map. 


\section{Conclusions}

Faced with the problem of insufficient materials in landslide emergencies, we proposed a novel USILD method in this paper. Employing high-resolution optical images obtained by satellites and airborne sensors, this method is the first to take advantage of visual salience to detect landslides by integrating the salience index based on the FT model and a LI based on reflective characteristics to produce the landslide risk map. Then, through morphological processing, a final refined landslide detection map is obtained. Generally speaking, the USILD method can well meet the requirements of landslide emergencies owing to its advantages of low data requirements, automaticity, and efficiency. In this study, we used the USILD method to investigate the Ludian landslide in 2014. An accurate landslide map was obtained with both the PA and UA at an acceptable level. From the risk map, the landslide risk area was estimated to be approximately $23.56 \mathrm{~km}^{2}$ for the Ludian landslide, which is close to the values in post-event official investigation reports. Also, the total time cost of the proposed method was $40 \mathrm{~s}$, reflecting its suitability for near real-time applications in landslide emergencies. We showed that our method can achieve higher accuracy and more automatic processing than other methods such as CDD, CDR, $k$-means, and SVM. The method requires no training samples and has a lower computational cost than supervised learning algorithms. Given its high detection accuracy and simple workflow, the proposed method is very promising for practical application in urban landslide emergency responses.

The proposed USILD method still has plenty of room for improvement. For example, by extracting more discriminant features to assist the landslide analysis, the detection accuracy can be further enhanced. The use of high-performance computing techniques can also greatly reduce the computing time when the study area and volume of data are large. In addition, we found that the proposed method has low performance for small landslides, with a relatively high omission rate. Maintenance of the shape and detail of landslides is also unsatisfactory. All of these issues will be addressed in future work.

\section{Acknowledgments}

This work was supported by the National Key R\&D Program of China (2018YFC0807005) and the China Engineering Science and Technology Project "Geographic Information Professional Knowledge Service System" (CKCEST-2020-1-5).

\section{References}

1 J. S. Kim and K. H. Kim: J. Appl. Remote Sens. 12 (2018) 3. https://doi.org/10.1080/13658810801914458

2 D. Petley: Geology 40 (2012) 10. https://doi.org/10.1130/G33217.1

3 C. F. Lee, W. K. Huang, Y. L. Chang, S. Y. Chi, and W. C. Liao: Geomorphology 300 (2018) 113. https://doi. org/10.1016/j.geomorph.2017.10.019

4 J. Nichol and M. S. Wong: Int. J. Remote Sens. 26 (2005) 9. https://doi.org/10.1080/01431160512331314047

5 G. R. Cochrane and G. H. Browne: Photogramm. Eng. Remote Sens. 47 (1981) 1205. https://www.asprs.org/ wp-content/uploads/pers/1981journal/aug/1981_aug_1205-1213.pdf

6 A. C. Mondini, M. Santangelo, M. Rocchetti, E. Rossetto, A. Manconi, and O. Monserrat: Remote Sens. 11 (2019) 7. https://doi.org/10.3390/rs11070760 
7 P. Lu, A. Stumpf, N. Kerle, and N.Casagli: IEEE Geosci. Remote Sens. Lett. 8 (2011) 4. http://doi.org/10.1109/ LGRS.2010.2101045

8 Z. Li, W. Shi, P. Lu, L. Yan, Q. Wang, and Z. Miao: Remote Sens. Environ. 187 (2016) 76. https://doi. org/10.1016/j.rse.2016.10.008

9 J. Uemoto, T. Moriyama, A. Nadai, S. Kojima, and T. Umehara: Nat. Hazards 95 (2019) 485. https://doi. org/10.1007/s11069-018-3492-8

10 A. L. Handwerger, S. Y. Jones, M. H. Huang, P. Amatya, H. R. Kerner, and D. B. Kirschbaum: Nat. Hazards Earth Syst. Sci. Discuss. (2020). https://doi.org/10.5194/nhess-2020-315

11 X. G. Shi, L. Zhang, C. Zhou, M. H. Li, and M. S. Liao: Landslides 15 (2018) 1015. https://doi.org/10.1007/ s10346-018-0975-3

12 W. C. Haneberg, W. F. Cole, and G. Kasali: Bulletin Eng. Geology Environ. 68 (2009) 2. https://oi. org/10.1007/s10064-009-0204-3

13 M. Ohki, T. Abe, T. Tadono, and M. Shimada: Earth Planets Space 72 (2020) 67. https://doi.org/10.1186/ s40623-020-01191-5

14 J. Barlow, Y. Martin, and S. E. Franklin: Can. J. Remote Sens. 29 (2003) 4. https://doi.org/10.5589/m03-018

15 S. Plank, A. Twele, and S. Martinis: Remote Sens. 8 (2016) 4. https://doi.org/10.3390/rs8040307

16 B. Pradhan, M. I. Seeni, and H. Nampak: Laser Scan. Appli. Landslide Assess. Springer. Cham. (2017) 69. https://doi.org/10.1007/978-3-319-55342-9_4

17 Y. Xu, D. L. George, J. Kim, Z. Lu, M. Riley, T. Griffin, and J. de la Fuente: Landslides (2020). https://doi. org/10.1007/s10346-020-01533-0

18 X. Li, X. Cheng, W. Chen, G. Chen, and S. Liu: Remote Sens. 7 (2015) 8. https://doi.org/10.3390/rs70809705

19 G. Cheng, L. Guo, T. Zhao, J. Han, H. Li, and J. Fang: Int. J. Remote Sens. 34 (2013) 1. https://doi.org/10.1080/ 01431161.2012 .705443

20 J. C. Wu and C. H. Chang: Proc. 2018 Springer, Cham. Conf. Arabian J. Geosci. (Springer, 2018) 155-158. https://doi.org/10.1007/978-3-030-01440-7_36

21 A. Ding, Q. Zhang, X. Zhou, and B. Dai: Proc. 2016 IEEE 31st Youth Academic Annual Conf. Chinese Associ. Autom. (IEEE, 2016) 444-448. http://doi.org/10.1109/YAC.2016.7804935

22 O. Ghorbanzadeh, T. Blaschke, K. Gholamnia, S. R. Meena, D. Tiede, and J. Aryal: Remote Sens. 11 (2019) 2. https://doi.org/10.3390/rs11020196

23 B. Yu, F. Chen, and C. Xu: Comput. Geosci. 135 (2020) 104388. https://doi.org/10.1016/j.cageo.2019.104388

24 K. He, J. Sun, and X. Tang: IEEE Trans. Pattern Analy. Mach. Intelli. 33 (2010) 12. http://doi.org/10.1109/ TPAMI.2010.168

25 R. Achanta, S. Hemami, F. Estrada, and S. Süsstrunk: Proc. 2009 IEEE Int Conf. Comput. Vis. Patt. Recog. (IEEE, 2009) 1597-1604. http://doi.org/10.1109/CVPR.2009.5206596

26 P. Soille and M. Pesaresi: IEEE Trans. Geosci. Remote Sens. 40 (2002) 9. http://doi.org/10.1109/ TGRS.2002.804618

27 V. Moosavi, A. Talebi, and B. Shirmohammadi: Geomorphology 204 (2014) 646. https://oi.org/10.1016/ j.geomorph.2013.09.012

28 T. R. Martha, N. Kerle, C. J. V. Westen, V. Jetten, and K. V. Kumar: ISPRS J Photogram. Remote Sens. 67 (2012) 105. https://doi.org/10.1016/j.isprsjprs.2011.11.004

29 Z. Y. Lv, W. Z. Shi, X. C. Zhou, and J. A. Benediktsson: Remote Sens. 9 (2017) 1112. https://doi.org/10.3390/ rs9111112

30 T. Celik: IEEE Geosci. Remote Sens. Lett. 6 (2009) 4. http://doi.org/10.1109/LGRS.2009.2025059

31 F. Guzzetti, P. Reichenbach, F. Ardizzone, M. Cardinali, and M. Galli: Geomorphology 81 (2006) 166. https:// doi.org/10.1016/j.geomorph.2006.04.007

\section{About the Authors}

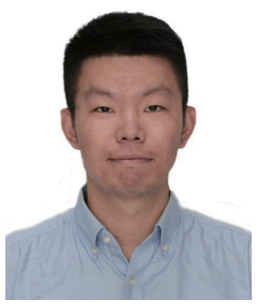

Xi Zhai received his B.S. degree in engineering of surveying and mapping from ShanDong Agricultural University, China, in 2006 and his M.S. and $\mathrm{Ph} . \mathrm{D}$. degrees in cartography and geographical information engineering from Wuhan University, China, in 2012 and 2017, respectively. Since 2017, he has been an engineer at National Geomatics Centre of China. His research interests are in emergency mapping, sensor webs, and spatial knowledge services. (zhaixi@ngcc.cn) 


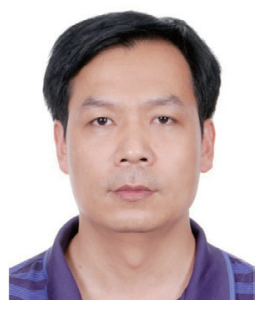

Wanzeng Liu received his B.S. degree in mine surveying, his M.S. degree in management science and engineering, and his $\mathrm{Ph} . \mathrm{D}$. degree in cartography and geographic information systems from China University of Mining and Technology in 1992, 2000, and 2005, respectively. From 1992 to 1997, he was an assistant engineer at Yongxia Mining Area Construction Management Commission, China. From 1997 to 2002, he was an engineer at Yongcheng Coal Power Group Co., China. Since 2006, he has been a senior engineer at National Geomatics Centre of China. His research interests are in emergency mapping, spatial knowledge services, and geographic information systems. (lwz@ngcc.cn)

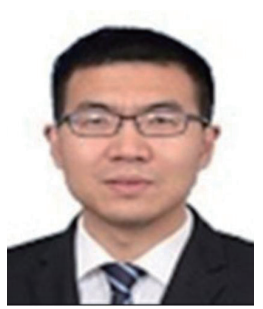

Chuan Yin received his B.E. degree from Sichuan Normal University, China, in 2009 and his M.S. and Ph.D. degrees from Capital Normal University, China, in 2012 and 2015, respectively. From 2015 to 2018, he did postdoctoral research at National Geomatics Centre of China. Since 2018, he has been a lecturer at Beijing University of Civil Engineering and Architecture. His research interests are in semantic webs and knowledge maps.

(yinchuan@bucea.edu.cn)

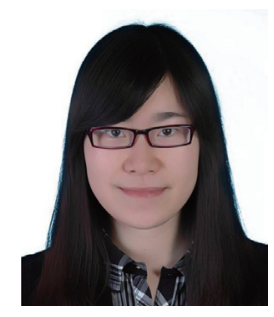

Yunlu Peng received her B.S. degree from Beijing University of Civil Engineering and Architecture, Beijing, in 2012. From 2012 to 2017, she was an assistant engineer at National Geomatics Centre of China. Since 2018, she has been an engineer at National Geomatics Centre of China. Her research interests are in geographic information systems. (pengyunlu@ngcc.cn)

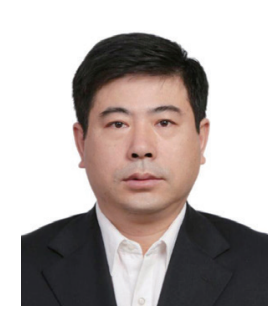

Yong Zhao received his B.S. degree in cartography from Wuhan Technical University of Surveying and Mapping, China, in 1989. From 1989 to 1992, he was an engineer at National Surveying and Mapping Resource Center of China. From 1992 to 2002, he was a manager at Beijing Yida Surveying and Mapping Technology Development Company, China. Since 2002, he has been a senior engineer at National Geomatics Centre of China. His research interests are digital cartography and GIS engineering technologies. (zhaoyong@ngcc.cn)

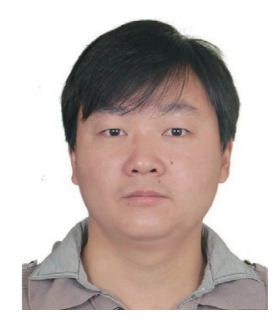

Ying Yang received his B.S. degree from Wuhan University, China, in 2007, his M.S. degree from the Chinese Academy of Sciences in 2010, and his Ph.D. degree from Wuhan University, China, in 2017. From 2010 to 2013, he was an assistant professor at Chinese Academy of Surveying and Mapping. Since 2017, he has been an engineer at National Geomatics Centre of China. His research interests are in photogrammetry, UAV systems, and sensors.

(yingy@whu.edu.cn) 


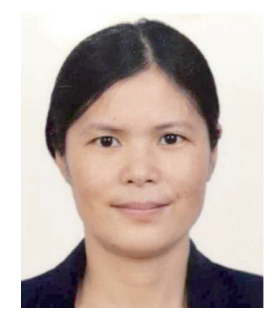

Xiuli Zhu received her B.S. degree from Wuhan Technical University of Surveying and Mapping and her M.S. degree from Wuhan University, China, in 1998 and 2001, respectively. Since 2001, she has been a senior engineer at National Geomatics Centre of China. Her research interests are in geoinformation services and emergency mapping. (zhuxiuli@ngcc.cn)

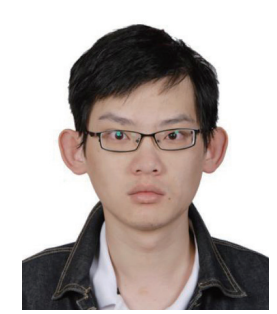

Ran Li received his B.S. and Ph.D. degrees from Wuhan University, China, in 2006 and 2012, respectively. Since 2012, he has been a senior engineer at National Geomatics Centre of China. His research interests are in photogrammetry and large-scale spatial data analysis. (liran@ngcc.cn)

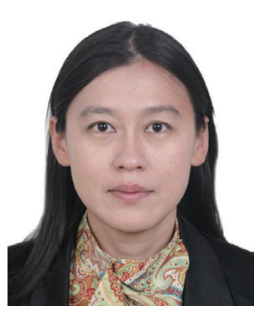

Tingting Zhao received her B.S. degree from Wuhan University, China, in 2000 and her M.S. degree from Chang'an University, Xi'an, China, in 2007. Since 2007, she has been a senior engineer at National Geomatics Centre of China. Her research interests are in thematic map compilation, surveying and mapping emergency support, and GIS development and applications. (zhaotingting@ngcc.cn) 